\title{
Bounds for the norm of lower triangular matrices on the Cesàro weighted sequence space
}

\section{Davoud Foroutannia* and Hadi Roopaei}

\section{"Correspondence:}

foroutan@vru.ac.ir

Department of Mathematics,

Vali-e-Asr University of Rafsanjan,

Rafsanjan, Iran

\section{Abstract}

This paper is concerned with the problem of finding bounds for the norm of lower triangular matrix operators from $l_{p}(w)$ into $c_{p}(W)$, where $c_{p}(w)$ is the Cesàro weighted sequence space and $\left(w_{n}\right)$ is a non-negative sequence. Also this problem is considered for lower triangular matrix operators from $c_{p}(W)$ into $I_{p}(W)$, and the norms of certain matrix operators such as Cesàro, Nörlund and weighted mean are computed.

Keywords: norm; lower triangular matrix; Nörlund matrix; weighted mean matrix; weighted sequence space

\section{Introduction}

Let $p \geq 1$ and $\omega$ denote the set of all real-valued sequences. The space $l_{p}$ is the set of all real sequences $x=\left(x_{n}\right) \in \omega$ such that

$$
\|x\|_{p}=\left(\sum_{n=1}^{\infty}\left|x_{n}\right|^{p}\right)^{1 / p}<\infty .
$$

If $w=\left(w_{n}\right) \in \omega$ is a non-negative sequence, we define the weighted sequence space $l_{p}(w)$ as follows:

$$
l_{p}(w):=\left\{x=\left(x_{n}\right) \in \omega: \sum_{n=1}^{\infty} w_{n}\left|x_{n}\right|^{p}<\infty\right\},
$$

with norm $\|\cdot\|_{p, w}$, which is defined in the following way:

$$
\|x\|_{p, w}=\left(\sum_{n=1}^{\infty} w_{n}\left|x_{n}\right|^{p}\right)^{1 / p} .
$$

Let $C=\left(c_{n, k}\right)$ denote the Cesàro matrix. We recall that the elements $c_{n, k}$ of the matrix $C$ are given by

$$
c_{n, k}= \begin{cases}\frac{1}{n} & \text { for } 1 \leq k \leq n, \\ 0 & \text { for } k>n .\end{cases}
$$


The sequence space defined by

$$
\begin{aligned}
c_{p}(w) & =\left\{\left(x_{n}\right) \in \omega: C x \in l_{p}(w)\right\} \\
& =\left\{\left(x_{n}\right) \in \omega: \sum_{n=1}^{\infty} w_{n}\left|\frac{1}{n} \sum_{i=1}^{n} x_{i}\right|^{p}<\infty\right\}
\end{aligned}
$$

is called the Cesàro weighted sequence space, and the norm $\|\cdot\|_{p, w, c}$ of the space is defined by

$$
\|x\|_{p, w, c}=\left(\sum_{n=1}^{\infty} w_{n}\left|\frac{1}{n} \sum_{i=1}^{n} x_{i}\right|^{p}\right)^{1 / p} .
$$

The Cesàro sequence spaces were studied in [1], where $w_{n}=1$ for all $n$. It is significant that in the special case $w_{n}=1$, we have $l_{p}(w)=l_{p}$ and $c_{p}(w)=c_{p}$.

Let $\left(w_{n}\right)$ be a non-negative sequence and $A=\left(a_{n, k}\right)$ be a lower triangular matrix with non-negative entries. In this paper, we shall consider the inequality of the form

$$
\|A x\|_{p, w, c} \leq U\|x\|_{p, w}
$$

and the inequality of the form

$$
\|A x\|_{p, w} \leq U\|x\|_{p, w, c}
$$

where $x=\left(x_{n}\right)$ is a non-negative sequence. The constant $U$ does not depend on $x$, and we seek the smallest possible value of $U$. We write $\|A\|_{p, w, c}$ for the norm of $A$ as an operator from $l_{p}(w)$ into $c_{p}(w),\|A\|_{p, c}$ for the norm of $A$ as an operator from $l_{p}$ into $c_{p},\|A\|_{c, p, w}$ for the norm of $A$ as an operator from $c_{p}(w)$ into $l_{p}(w),\|A\|_{c, p}$ for the norm of $A$ as an operator from $c_{p}$ into $l_{p},\|A\|_{p, w}$ for the norm of $A$ as an operator from $l_{p}(w)$ into itself and $\|A\|_{p}$ for the norm of $A$ as an operator from $l_{p}$ into itself.

The problem of finding the norm of a lower triangular matrix on the sequence spaces $l_{p}$ and $l_{p}(w)$ has been studied before in [2-8]. In the study, we will expand this problem for matrix operators from $l_{p}(w)$ into $c_{p}(w)$ and matrix operators from $c_{p}(w)$ into $l_{p}(w)$, and we consider certain matrix operators such as Cesàro, Nörlund and weighted mean. The study is an extension of some results obtained by $[3,7]$.

\section{The norm of matrix operators from $I_{p}(w)$ into $c_{p}(w)$}

In this section, we tend to compute the bounds for the norm of lower triangular matrix operators from $l_{p}(w)$ into $c_{p}(w)$. In particular, we apply our results for lower triangular matrix operators from $l_{p}$ into $c_{p}$, when $w_{n}=1$ for all $n$.

Throughout this paper, let $A=\left(a_{n, k}\right)$ be a matrix with non-negative real entries i.e., $a_{n, k} \geq 0$, for all $n, k$. This implies that $\|A x\|_{p, w, c} \leq\|A|x|\|_{p, w, c}$, and hence the non-negative sequences are sufficient to determine the norm of $A$. We say that $A=\left(a_{n, k}\right)$ is lower triangular if $a_{n, k}=0$ for $n<k$. A non-negative lower triangular matrix is called a summability matrix if $\sum_{k=1}^{n} a_{n, k}=1$ for all $n$.

We first state some lemmas from $[3,7]$, which are needed for our main result. Set $\xi^{+}=$ $\max (\xi, 0)$ and $\xi^{-}=\min (\xi, 0)$ and $p^{*}=p /(p-1)$. 
Lemma 2.1 ([3], Lemma 2.1) Let $a$ and $x$ be two non-negative sequences, then for all n,

$$
\sum_{k=1}^{n} a_{k} x_{k} \leq\left\{\max _{1 \leq k \leq n} \frac{1}{n-k+1} \sum_{j=k}^{n} x_{j}\right\} \sum_{k=1}^{n}(n-k+1)\left(a_{k}-a_{k-1}\right)^{+} .
$$

Lemma 2.2 ([3], Lemma 2.2) Let $N \geq 1$, and let $a$ and $x$ be two non-negative sequences. If $x_{N} \geq x_{N+1} \geq \cdots \geq 0$ and $x_{n}=0$ for $n<N$, then

$$
\sum_{k=1}^{n} a_{k} x_{k} \geq\left(\frac{1}{n} \sum_{j=1}^{n} x_{j}\right)\left\{n a_{N}+\frac{n}{n-N+1} \sum_{k=N+1}^{n}(n-k+1)\left(a_{k}-a_{k-1}\right)^{-}\right\}
$$

for all $n$.

Lemma 2.3 ([7], Lemma 1.4) Let $p>1$ and $w=\left(w_{n}\right)$ be a decreasing sequence with nonnegative entries and $\sum_{n=1}^{\infty} \frac{w_{n}}{n}$ be divergent. Let $N \geq 1$ and the matrix $C_{N}=\left(c_{n, k}^{N}\right)$ be with the following entries:

$$
c_{n, k}^{N}= \begin{cases}\frac{1}{n+N-1} & \text { for } n \geq k \\ 0 & \text { for } n<k\end{cases}
$$

Then $\left\|C_{N}\right\|_{p, w}$ is determined by non-negative decreasing sequences and $\left\|C_{N}\right\|_{p, w}=p^{*}$.

Note that $C_{1}$ is the well-known Cesàro matrix.

Lemma 2.4 ([7], Lemma 1.5) If $p>1$ and $x$ and $w$ are two non-negative sequences and also $w$ is decreasing, then

$$
\sum_{j=1}^{\infty} w_{j} \max _{1 \leq i \leq j}\left(\frac{1}{j-i+1} \sum_{k=i}^{j} x_{k}\right)^{p} \leq\left(p^{*}\right)^{p} \sum_{k=1}^{\infty} w_{k} x_{k}^{p}
$$

We set $a_{0,0}=0$ and $a_{n, 0}=0$ for $n \geq 1$ and

$$
\begin{aligned}
& M_{A}=\sup _{n \geq 1}\left\{\sum_{k=1}^{n} \frac{n-k+1}{n}\left(\sum_{i=k}^{n} a_{i, k}-\sum_{i=k-1}^{n} a_{i, k-1}\right)^{+}\right\}, \\
& m_{A}=\sup _{N \geq 1} \inf _{n \geq N}\left\{\sum_{i=N}^{n} a_{i, N}+\frac{1}{n-N+1} \sum_{k=N+1}^{n}(n-k+1)\left(\sum_{i=k}^{n} a_{i, k}-\sum_{i=k-1}^{n} a_{i, k-1}\right)^{-}\right\} .
\end{aligned}
$$

We are now ready to present the main result of this section.

Theorem 2.5 Suppose that $p>1$ and $w=\left(w_{n}\right)$ is a decreasing sequence with non-negative entries. If $A=\left(a_{n, k}\right)$ is a lower triangular matrix with non-negative entries, then we have the following statements.

(i) $\|A\|_{p, w, c} \leq p^{*} M_{A}$. Moreover, if $M_{A}<\infty$, then $A$ is a bounded matrix operator from $l_{p}(w)$ into $c_{p}(w)$.

(ii) If $\sum_{n=1}^{\infty} \frac{w_{n}}{n}$ is divergent and $\left(\frac{w_{n}}{w_{n+1}}\right)$ is decreasing, then $\|A\|_{p, w, c} \geq p^{*} m_{A}$. 
Therefore if $w=\left(w_{n}\right)$ is a decreasing sequence with non-negative entries and $\left(\frac{w_{n}}{w_{n+1}}\right)$ is decreasing and $\sum_{n=1}^{\infty} \frac{w_{n}}{n}=\infty$, then

$$
p^{*} m_{A} \leq\|A\|_{p, w, c} \leq p^{*} M_{A}
$$

In particular, if $w_{n}=1$ for all $n$ and if $M_{A}<\infty$, then $A$ is a bounded matrix operator from $l_{p}$ into $c_{p}$ and $p^{*} m_{A} \leq\|A\|_{p, c} \leq p^{*} M_{A}$.

Proof (i) Let $\left(x_{n}\right)$ be a non-negative sequence. By using Lemma 2.1, we get

$$
\begin{aligned}
& \sum_{k=1}^{n}\left(\frac{1}{n} \sum_{i=k}^{n} a_{i, k}\right) x_{k} \\
& \quad \leq\left\{\max _{1 \leq k \leq n} \frac{1}{n-k+1} \sum_{j=k}^{n} x_{j}\right\} \sum_{k=1}^{n} \frac{n-k+1}{n}\left(\sum_{i=k}^{n} a_{i, k}-\sum_{i=k-1}^{n} a_{i, k-1}\right)^{+} \\
& \quad \leq M_{A} \max _{1 \leq k \leq n}\left\{\frac{1}{n-k+1} \sum_{j=k}^{n} x_{j}\right\} .
\end{aligned}
$$

By applying Lemma 2.4, we deduce that

$$
\begin{aligned}
\sum_{n=1}^{\infty} w_{n}\left(\sum_{k=1}^{n}\left(\frac{1}{n} \sum_{i=k}^{n} a_{i, k}\right) x_{k}\right)^{p} & \leq M_{A}^{p} \sum_{n=1}^{\infty} w_{n} \max _{1 \leq k \leq n}\left(\frac{1}{n-k+1} \sum_{j=k}^{n} x_{j}\right)^{p} \\
& \leq\left(p^{*} M_{A}\right)^{p} \sum_{k=1}^{\infty} w_{k} x_{k}^{p} .
\end{aligned}
$$

(ii) We have $m_{A}=\sup _{N \geq 1} \beta_{N}$, where

$$
\beta_{N}=\inf _{n \geq N}\left\{\sum_{i=N}^{n} a_{i, N}+\frac{1}{n-N+1} \sum_{k=N+1}^{n}(n-k+1)\left(\sum_{i=k}^{n} a_{i, k}-\sum_{i=k-1}^{n} a_{i, k-1}\right)^{-}\right\} .
$$

Let $N \geq 1$, so that $\beta_{N} \geq 0$. If $y=\left(y_{n}\right)$ is a decreasing sequence with non-negative entries and $\|y\|_{p, w}=1$, we set $x_{1}=x_{2}=\cdots=x_{N-1}=0$ and

$$
x_{n+N-1}=\left(\frac{w_{n}}{w_{n+N-1}}\right)^{1 / p} y_{n}
$$

for all $n \geq 1$. So $\|x\|_{p, w}=\|y\|_{p, w}=1$, and from Lemma 2.2 it follows that

$$
\begin{aligned}
\|A\|_{p, w, c}^{p} & \geq \sum_{n=1}^{\infty} w_{n}\left(\sum_{k=1}^{n}\left(\frac{1}{n} \sum_{i=k}^{n} a_{i, k}\right) x_{k}\right)^{p} \\
& \geq \beta_{N}^{p} \sum_{n=1}^{\infty} w_{n}\left(\frac{1}{n} \sum_{j=1}^{n} x_{j}\right)^{p} \\
& =\beta_{N}^{p} \sum_{n=1}^{\infty} w_{n+N-1}\left(\frac{1}{n+N-1} \sum_{j=1}^{n} x_{j+N-1}\right)^{p}
\end{aligned}
$$




$$
\begin{aligned}
& =\beta_{N}^{p} \sum_{n=1}^{\infty} w_{n+N-1}\left(\frac{1}{n+N-1} \sum_{j=1}^{n}\left(\frac{w_{j}}{w_{j+N-1}}\right)^{1 / p} y_{j}\right)^{p} \\
& \geq \beta_{N}^{p}\left\|C_{N} y\right\|_{p, w,}^{p} .
\end{aligned}
$$

By Lemma 2.3, we conclude that $\|A\|_{p, w, c} \geq p^{*} \beta_{N}$, so

$$
\|A\|_{p, w, c} \geq p^{*} m_{A} .
$$

In what follows we assume that $w=\left(w_{n}\right)$ is a decreasing sequence with non-negative entries and $\left(\frac{w_{n}}{w_{n+1}}\right)$ is decreasing and $\sum_{n=1}^{\infty} \frac{w_{n}}{n}=\infty$.

At first we bring a corollary of Theorem 2.5 for a lower triangular matrix $A=\left(a_{n, k}\right)$. The rows of $C_{1} A$ are increasing, where $C_{1}$ is the Cesàro matrix and

$$
\left(C_{1} A\right)_{n, k}=\sum_{i=1}^{\infty} c_{n, i}^{1} a_{i, k}=\frac{1}{n} \sum_{i=k}^{n} a_{i, k}, \quad(n, k=1,2, \ldots) .
$$

Corollary 2.6 Suppose that $p>1$ and $A=\left(a_{n, k}\right)$ is a non-negative lower triangular matrix that $\sum_{i=k-1}^{n} a_{i, k-1} \leq \sum_{i=k}^{n} a_{i, k}$ for $1<k \leq n$. Then

$$
\|A\|_{p, w, c}=p^{*} \sup _{n \geq 1} a_{n, n} .
$$

In particular, $\|I\|_{p, w, c}=p^{*}$, where I is the identity matrix.

Proof Since the finite sequence $\left(\sum_{i=k}^{n} a_{i, k}\right)_{k=1}^{n}$ is increasing for each $n$, we have

$$
\left(\sum_{i=k}^{n} a_{i, k}-\sum_{i=k-1}^{n} a_{i, k-1}\right)^{+}=\sum_{i=k}^{n} a_{i, k}-\sum_{i=k-1}^{n} a_{i, k-1}
$$

for $1 \leq k \leq n$. Hence

$$
\begin{aligned}
M_{A} & =\sup _{n \geq 1}\left\{\sum_{k=1}^{n} \frac{n-k+1}{n}\left(\sum_{i=k}^{n} a_{i, k}-\sum_{i=k-1}^{n} a_{i, k-1}\right)\right\} \\
& =\sup _{n \geq 1} \frac{1}{n} \sum_{k=1}^{n} \sum_{i=k}^{n} a_{i, k} \leq \sup _{n \geq 1} a_{n, n} .
\end{aligned}
$$

Moreover,

$$
\left(\sum_{i=k}^{n} a_{i, k}-\sum_{i=k-1}^{n} a_{i, k-1}\right)^{-}=0 \quad(1 \leq k \leq n)
$$

and

$$
m_{A}=\sup _{N \geq 1} \inf _{n \geq N} \sum_{i=N}^{n} a_{i, N}=\sup _{n \geq 1} a_{n, n} .
$$

Hence, according to Theorem 2.5, we obtain the desired result. 
Example 2.7 Let $A=\left(a_{n, k}\right)$ be defined by

$$
a_{n, k}= \begin{cases}\frac{1}{n^{2}} & \text { for } k<n \\ \frac{2 n-1}{n} & \text { for } k=n \\ 0 & \text { for } k>n\end{cases}
$$

Since the finite sequence $\left(\sum_{i=k}^{n} a_{i, k}\right)_{k=1}^{n}$ is increasing for each $n$ and $\sup _{n \geq 1} a_{n, n}=2$, by Corollary 2.6, we have $\|A\|_{p, w, c}=2 p^{*}$.

Now, in the second case, we state some corollaries of Theorem 2.5 for a lower triangular matrix $A$, where the rows of $C_{1} A$ are decreasing.

Corollary 2.8 Suppose that $p>1$ and $A=\left(a_{n, k}\right)$ is a lower triangular matrix with $\sum_{i=k-1}^{n} a_{i, k-1} \geq \sum_{i=k}^{n} a_{i, k}$ for $1<k \leq n$. Then

$$
p^{*}\left(\inf _{n \geq 1} \frac{1}{n} \sum_{k=1}^{n} \sum_{i=k}^{n} a_{i, k}\right) \leq\|A\|_{p, w, c} \leq p^{*}\left(\sup _{n \geq 1} \sum_{i=1}^{n} a_{i, 1}\right) .
$$

In particular, for summability matrices the left-hand side of the above inequality reduces to $p^{*}$.

Moreover, if the right-hand side of the above inequality is finite, then $A$ is a bounded matrix operator from $l_{p}(w)$ into $c_{p}(w)$.

Proof Since the finite sequence $\left(\sum_{i=k}^{n} a_{i, k}\right)_{k=1}^{n}$ is decreasing for each $n$, we have

$$
\left(\sum_{i=k}^{n} a_{i, k}-\sum_{i=k-1}^{n} a_{i, k-1}\right)^{+}=0 \quad(1<k \leq n)
$$

and $\left(\sum_{i=1}^{n} a_{i, 1}-\sum_{i=0}^{n} a_{i, 0}\right)^{+}=\sum_{i=1}^{n} a_{i, 1}$. Hence $M_{A}=\sup _{n \geq 1} \sum_{i=1}^{n} a_{i, 1}$. Moreover,

$$
\left(\sum_{i=k}^{n} a_{i, k}-\sum_{i=k-1}^{n} a_{i, k-1}\right)^{-}=\sum_{i=k}^{n} a_{i, k}-\sum_{i=k-1}^{n} a_{i, k-1}
$$

for $1<k \leq n$, so

$$
\begin{aligned}
m_{A} & =\sup _{N \geq 1} \inf _{n \geq N}\left\{\sum_{i=N}^{n} a_{i, N}+\frac{1}{n-N+1} \sum_{k=N+1}^{n}(n-k+1)\left(\sum_{i=k}^{n} a_{i, k}-\sum_{i=k-1}^{n} a_{i, k-1}\right)\right\} \\
& =\sup _{N \geq 1} \inf _{n \geq N} \frac{1}{n-N+1} \sum_{k=N}^{n} \sum_{i=k}^{n} a_{i, k} \\
& \geq \inf _{n \geq 1} \frac{1}{n} \sum_{k=1}^{n} \sum_{i=k}^{n} a_{i, k} .
\end{aligned}
$$

Therefore, by Theorem 2.5, we prove the desired result.

The two examples of Corollary 2.8 are given as follows. 
Example 2.9 Suppose that $\alpha \geq 2$ and the matrix $A=\left(a_{n, k}\right)$ is defined by

$$
a_{n, k}= \begin{cases}\frac{1}{n^{\alpha}} & \text { for } n \geq k \\ 0 & \text { for } n<k\end{cases}
$$

Since $\sum_{i=k}^{n} a_{i, k}=\sum_{i=k}^{n} \frac{1}{i^{\alpha}}$ and $\sum_{i=k-1}^{n} a_{i, k-1} \geq \sum_{i=k}^{n} a_{i, k}$ for $1<k \leq n$, we have $0 \leq\|A\|_{p, w, c} \leq$ $p^{*} \zeta(\alpha)$, where $\zeta(\alpha)=\sum_{n=1}^{\infty} \frac{1}{n^{\alpha}}$.

Example 2.10 Suppose that the matrix $A=\left(a_{n, k}\right)$ is defined by

$$
a_{n, k}= \begin{cases}\frac{1}{n(n+1)} & \text { for } n \geq k \\ 0 & \text { for } n<k\end{cases}
$$

Since $\sum_{i=k}^{n} a_{i, k}=\sum_{i=k}^{n} \frac{1}{i(i+1)}$, by Corollary 2.8 , we have $0 \leq\|A\|_{p, w, c} \leq p^{*}$.

We apply the above corollary to the following two special cases.

Let $\left(a_{n}\right)$ be a non-negative sequence with $a_{1}>0$, and $A_{n}=a_{1}+\cdots+a_{n}$. The Nörlund matrix $N_{a}=\left(a_{n, k}\right)$ is defined as follows:

$$
a_{n, k}= \begin{cases}\frac{a_{n-k+1}}{A_{n}} & \text { for } 1 \leq k \leq n, \\ 0 & \text { for } k>n\end{cases}
$$

Also the weighted mean matrix $M_{a}=\left(a_{n, k}\right)$ is defined by

$$
a_{n, k}= \begin{cases}\frac{a_{k}}{A_{n}} & \text { for } 1 \leq k \leq n, \\ 0 & \text { for } k>n\end{cases}
$$

Corollary 2.11 Suppose that $p>1$ and $N_{a}=\left(a_{n, k}\right)$ is the Nörlund matrix and $\left(a_{n}\right)$ is an increasing sequence. Then

$$
p^{*} \leq\left\|N_{a}\right\|_{p, w, c} \leq p^{*}\left(\sup _{n \geq 1} \sum_{i=1}^{n} \frac{a_{i}}{A_{i}}\right) .
$$

Proof Since $N_{a}$ is a summability matrix and $\sum_{i=1}^{n} a_{i, 1}=\sum_{i=1}^{n} \frac{a_{i}}{A_{i}}$, by applying Corollary 2.8, we have the desired result.

Corollary 2.12 Suppose that $p>1$ and $M_{a}=\left(a_{n, k}\right)$ is the weighted mean matrix and $\left(a_{n}\right)$ is a decreasing sequence. Then

$$
p^{*} \leq\left\|M_{a}\right\|_{p, w, c} \leq p^{*} a_{1}\left(\sup _{n \geq 1} \sum_{i=1}^{n} \frac{1}{A_{i}}\right) .
$$

Proof Since $M_{a}$ is a summability matrix and $\sum_{i=1}^{n} a_{i, 1}=\sum_{i=1}^{n} \frac{a_{1}}{A_{i}}$, by Corollary 2.8 , the proof is obvious.

Finally, in the third case, if the rows of $C_{1} A$ are neither increasing nor decreasing, we present the following theorem. 
Theorem 2.13 Suppose that $p>1$ and $A=\left(a_{n, k}\right)$ is a non-negative lower triangularmatrix. If $A$ is a bounded matrix operator from $l_{p}(w)$ into itself, then $A$ is a bounded matrix operator from $l_{p}(w)$ into $c_{p}(w)$ and

$$
\|A\|_{p, w, c} \leq p^{*}\|A\|_{p, w} .
$$

Proof We have

$$
\begin{aligned}
\|A x\|_{p, w, c}^{p} & =\sum_{n=1}^{\infty} w_{n}\left|\frac{1}{n} \sum_{k=1}^{n} \sum_{j=1}^{k} a_{k, j} x_{j}\right|^{p} \\
& =\sum_{n=1}^{\infty} w_{n}\left|\sum_{j=1}^{n}\left(C_{1} A\right)_{n, j} x_{j}\right|^{p}=\left\|\left(C_{1} A\right) x\right\|_{p, w}^{p} .
\end{aligned}
$$

Hence, by Lemma 2.3, we conclude that $\|A\|_{p, w, c}=\left\|C_{1} A\right\|_{p, w} \leq p^{*}\|A\|_{p, w}$.

We apply the above theorem to the following two Nörlund and weighted mean matrices.

Corollary 2.14 ([7], Corollary 1.3) Suppose that $p>1$ and $N_{a}=\left(a_{n, k}\right)$ is the Nörlund matrix and $\left(a_{n}\right)$ is a decreasing sequence with $a_{n} \downarrow \alpha$ and $\alpha>0$. Then

$$
\left\|N_{a}\right\|_{p, w}=p^{*}
$$

Corollary 2.15 Suppose that $p>1$ and $N_{a}=\left(a_{n, k}\right)$ is the Nörlund matrix and $\left(a_{n}\right)$ is a decreasing sequence with $a_{n} \downarrow \alpha$ and $\alpha>0$. Then

$$
\left\|N_{a}\right\|_{p, w, c} \leq\left(p^{*}\right)^{2} \text {. }
$$

Proof By applying Theorem 2.13 and Corollary 2.14, we have the desired result.

Corollary 2.16 ([7], Corollary 1.4) Suppose that $p>1$ and $M_{a}=\left(a_{n, k}\right)$ is the weighted mean matrix and $\left(a_{n}\right)$ is an increasing sequence with $a_{n} \uparrow \alpha$ and $\alpha<\infty$. Then

$$
\left\|M_{a}\right\|_{p, w}=p^{*} .
$$

Corollary 2.17 Suppose that $p>1$ and $M_{a}=\left(a_{n, k}\right)$ is the weighted mean matrix and $\left(a_{n}\right)$ is an increasing sequence with $a_{n} \uparrow \alpha$ and $\alpha<\infty$. Then

$$
\left\|M_{a}\right\|_{p, w, c} \leq\left(p^{*}\right)^{2} .
$$

Proof By using Theorem 2.13 and Corollary 2.16, the proof is clear.

\section{The norm of matrix operators from $c_{p}(w)$ into $I_{p}(w)$}

In this section, we compute the bounds for the norm of lower triangular matrix operators from $c_{p}(w)$ into $l_{p}(w)$. In particular, when $w_{n}=1$ for all $n$, the bounds for the norm of lower triangular matrix operators from $c_{p}$ into $l_{p}$ are deduced. Moreover, we apply our results for Cesàro, Nörlund and weighted mean matrices.

We begin with a proposition which is needed to prove the main theorem of this section. 
Proposition 3.1 ([6], Proposition 5.1). Let $p>1$ and $w=\left(w_{n}\right)$ be a decreasing sequence with non-negative entries, and let $C_{1}$ be the Cesàro matrix. Then we have $\left\|C_{1}\right\|_{p, w} \leq p^{*}$.

Theorem 3.2 Suppose that $p>1$ and $w=\left(w_{n}\right)$ is a sequence with non-negative entries and $A=\left(a_{n, k}\right)$ is a lower triangular matrix with non-negative entries. We have

$$
\frac{1}{p^{*}}\|A\|_{p, w} \leq\|A\|_{c, p, w} \leq \sup _{n \geq 1}\left(n \sup _{1 \leq k \leq n} a_{n, k}\right) .
$$

Moreover, if the right-hand side of the above inequality is finite, then $A$ is a bounded matrix operator from $c_{p}(w)$ into $l_{p}(w)$. In particular, if $w_{n}=1$ for all $n$, then we have

$$
\frac{1}{p^{*}}\|A\|_{p} \leq\|A\|_{c, p} \leq \sup _{n \geq 1}\left(n \sup _{1 \leq k \leq n} a_{n, k}\right) .
$$

Proof Suppose that $x \in c_{p}(w)$

$$
\begin{aligned}
\|A x\|_{p, w}^{p} & =\sum_{n=1}^{\infty} w_{n}\left|\sum_{k=1}^{n} a_{n, k} x_{k}\right|^{p} \\
& \leq \sum_{n=1}^{\infty} w_{n}\left(\sup _{1 \leq k \leq n} a_{n, k} \sum_{k=1}^{n} x_{k}\right)^{p} \\
& \leq \sup _{n \geq 1}\left(n \sup _{1 \leq k \leq n} a_{n, k}\right)^{p} \sum_{n=1}^{\infty} w_{n}\left(\frac{1}{n} \sum_{k=1}^{n} x_{k}\right)^{p} \\
& =\sup _{n \geq 1}\left(n \sup _{1 \leq k \leq n} a_{n, k}\right)^{p}\|x\|_{p, w, c}^{p} .
\end{aligned}
$$

Hence

$$
\frac{\|A x\|_{p, w}}{\|x\|_{p, w, c}} \leq \sup _{n \geq 1}\left(n \sup _{1 \leq k \leq n} a_{n, k}\right)
$$

and

$$
\|A\|_{c, p, w} \leq \sup _{n \geq 1}\left(n \sup _{1 \leq k \leq n} a_{n, k}\right)
$$

On the other hand, Proposition 3.1 concludes that $\|x\|_{p, w, c}=\left\|C_{1} x\right\|_{p, w} \leq p^{*}\|x\|_{p, w}$, so

$$
\frac{\|A x\|_{p, w}}{\|x\|_{p, w, c}} \geq \frac{1}{p^{*}} \frac{\|A x\|_{p, w}}{\|x\|_{p, w}} .
$$

Therefore $\frac{1}{p^{*}}\|A\|_{p, w} \leq\|A\|_{c, p, w}$, and the proof is complete.

Corollary 3.3 If $p>1$, then the generalized Cesàro matrix $C_{N}$ is bounded from $c_{p}(w)$ into $l_{p}(w)$ and

$$
\left\|C_{N}\right\|_{c, p, w}=1
$$


Proof Since

$$
\sup _{n \geq 1}\left(n \sup _{1 \leq k \leq n} a_{n, k}\right)=\sup _{n \geq 1} \frac{n}{n+N-1}=1,
$$

by using Lemma 2.3 and Theorem 3.2, the proof is obvious.

We apply the above theorem to the following two special cases.

Corollary 3.4 Suppose that $p>1$ and $N_{a}=\left(a_{n, k}\right)$ is the Nörlund matrix and $\left(a_{n}\right)$ is a decreasing sequence with $a_{n} \downarrow \alpha$ and $\alpha>0$. Then

$$
1 \leq\left\|N_{a}\right\|_{c, p, w} \leq a_{1} \sup _{n \geq 1} \frac{n}{A_{n}} .
$$

Proof By Theorem 3.2 and Corollary 2.14, the proof is clear.

Example 3.5 Let $\alpha>0$ and $a_{n}=\alpha+\frac{1}{n^{\alpha+1}}$ for all $n$. The sequence $\left(a_{n}\right)$ is decreasing and $a_{n} \downarrow \alpha$ and also $a_{1} \sup _{n \geq 1} \frac{n}{A_{n}}=1+\frac{1}{\alpha}$. So

$$
1 \leq\left\|N_{a}\right\|_{c, p, w} \leq 1+\frac{1}{\alpha}
$$

Specially $\left\|N_{a}\right\|_{c, p, w} \rightarrow 1$, when $\alpha \rightarrow \infty$.

Corollary 3.6 Suppose that $p>1$ and $M_{a}=\left(a_{n, k}\right)$ is the weighted mean matrix and $\left(a_{n}\right)$ is an increasing sequence with $a_{n} \uparrow \alpha$ and $\alpha<\infty$. Then

$$
1 \leq\left\|M_{a}\right\|_{c, p, w} \leq \sup _{n \geq 1} \frac{n a_{n}}{A_{n}} .
$$

Proof By using Theorem 3.2 and Corollary 2.16, the proof is obvious.

Example 3.7 Let $a_{n}=1-\frac{1}{(n+1)^{2}}$ for all $n$. The sequence $\left(a_{n}\right)$ is increasing and $a_{n} \uparrow 1$ and also

$$
\sup _{n \geq 1} \frac{n a_{n}}{A_{n}}=\frac{3 a_{3}}{A_{3}} \simeq 1.091
$$

So

$$
1 \leq\left\|M_{a}\right\|_{c, p, w} \leq 1.091
$$

\section{Conclusions}

In the present study, we considered the problem of finding bounds for the norm of lower triangular matrix operators from $l_{p}(w)$ into $c_{p}(w)$ and from $c_{p}(w)$ into $l_{p}(w)$. Moreover, we computed the norms of certain matrix operators such as Cesàro, Nörlund and weighted mean, and we extended some results of $[3,7]$. 
Authors' contributions

All authors contributed equally to the writing of this paper. All authors read and approved the final manuscript.

\section{Acknowledgements}

The second author would like to record his pleasure to Dr. Gholareza Talebi [Department of Mathematics, Faculty of Sciences, Vali-E-Asr University of Rafsanjan] for his valuable conversations during the preparation of the present paper.

\section{Publisher's Note}

Springer Nature remains neutral with regard to jurisdictional claims in published maps and institutional affiliations.

Received: 22 December 2016 Accepted: 22 March 2017 Published online: 04 April 2017

\section{References}

1. Ng, P-N, Lee, P-Y: Cesàro sequence spaces of non-absolute type. Comment. Math. Prace Mat. 20(2), $429-433$ (1978)

2. Grahame, B: Factorizing the Classical Inequalities, vol. 576. Am. Math. Soc., Providence (1996)

3. Chen, C-P, Luor, D-C, Ou, Z-Y: Extensions of Hardy inequality. J. Math. Anal. Appl. 273(1), 160-171 (2002)

4. Hardy, GH: An inequality for Hausdorff means. J. Lond. Math. Soc. 1(1), 46-50 (1943)

5. Hardy, GH, Littlewood, JE, Pólya, G: Inequalities, 2nd edn. (1934)

6. Jameson, GJO, Lashkaripour, R: Norms of certain operators on weighted lp spaces and Lorentz sequence spaces. JIPAM. J. Inequal. Pure Appl. Math. 3 1, (2002)

7. Lashkaripour, R: Extension of Hardy inequality on weighted sequence spaces. J. Sci. Islam. Repub. Iran 20 2, (2009)

8. Pečarić, J, Perić, I, Roki, R: On bounds for weighted norms for matrices and integral operators. Linear Algebra Appl. 326(1-3), 121-135 (2001)

\section{Submit your manuscript to a SpringerOpen ${ }^{\circ}$ journal and benefit from:}

- Convenient online submission

- Rigorous peer review

Immediate publication on acceptance

Open access: articles freely available online

- High visibility within the field

- Retaining the copyright to your article 